\title{
Development of tailor-made silica fibres for TL dosimetry
}

\begin{abstract}
The Ge dopant in commercially available silica optical fibres gives rise to appreciable thermoluminscence (TL), weight-for-weight offering sensitivity to MV X-rays several times that of the LiF dosimeter TLD100. The response of these fibres to UV radiation, X-rays, electrons, protons, neutrons and alpha particles, with doses from a fraction of 1 Gy up to 10 $\mathrm{kGy}$, have stimulated further investigation of the magnitude of the TL signal for intrinsic and doped SiO2 fibres. We represent a consortium effort between Malaysian partners and the University of Surrey, aimed at production of silica fibres with specific TL dosimetry applications, utilizing modified chemical vapour deposition (MCVD) doped silicaï glass production and fibre-pulling facilities. The work is informed by defect and dopant concentration and various production dependences including pulling parameters such as temperature, speed and tension; the fibres also provide for spatial resolutions down to $<10$ $\mu \mathrm{m}$, confronting many limitations faced in use of conventional (TL) dosimetry. Early results are shown for high spatial resolution $(\sim 0.1 \mathrm{~mm})$ single-core Ge-doped TL sensors, suited to radiotherapy applications. Preliminary results are also shown for undoped flat optical fibres of $\mathrm{mm}$ dimensions and Ge-B doped flat optical fibres of sub-mm dimensions, with potential for measurement of doses in medical diagnostic applications.
\end{abstract}

Keyword: Silica fibres; Defects; Thermoluminescence; Dosimetry 